\title{
Intelligent Advisory System for Designing Plastics Products
}

\author{
U. Sancin ${ }^{1}$ and B. Dolšak ${ }^{2}$
}

\begin{abstract}
Plastics product design is very experience dependent process. In spite of various computer tools available on the market, the designer has to rely on personal or supporting experts' knowledge and experience when designing plastics products. Proposed development of the intelligent advisory system presented in this paper involves two methodologies. "Design for X" strategy will be applied to consider specific design aspects for plastic products, while "Knowledge-Based Engineering" will be used for knowledge acquisition, its systematization and utilization within the system. The major benefit of the intelligent support provided by the advisory system will be faster and more reliable product development process, as the system will offer the user some recommendation and advice about material selection and related production process. Thus, the expert team could be contracted. Minimized development process costs along with optimal technical design solutions for plastic products will enable small and medium size enterprises to compete with their plastics products on the global market.
\end{abstract}

${ }^{1}$ U. Sancin, BSc

Faculty of Mechanical Engineering, University of Maribor, SI-2000 Maribor, Slovenia, email: urska.sancin@uni-mb.si

${ }^{2}$ Assoc. Prof. Dr. B. Dolšak

Faculty of Mechanical Engineering, University of Maribor, SI-2000 Maribor, Slovenia, email: dolsak@uni-mb.si

Please use the following format when citing this chapter: 


\section{Introduction}

Not long ago, Computer Aided Design (CAD) represented quite revolutionary approach in both, computer and engineering science. Nowadays designers have at their disposal a wide range of CAD applications like drafting, modelling, analysing, simulation, etc. Using CAD tools design is much more effective. Existing CAD is almost perfected on the graphic presentation of design but still has serious limitations at providing recommendation and advice to the designer. Information like material, surface treatment, tolerances, etc. are generally represented as remarks on the drawing or by attaching attributes to a three-dimensional model while only geometric form and dimensions are covered satisfactory.

However, CAD applications are reality in modern engineering design process and working without them is almost impossible to imagine. As mentioned before, the existing computer support does not offer adequate information or advice to designer when dealing with dilemmas about material selection or process selection etc. Therefore designer has to take a decision based on his or her own knowledge and experience. As one person cannot master such extensive range of knowledge to take all the correct engineering decisions, the designer has to collaborate with a group of experts with different expertises.

The main objective of the proposed research presented in this paper is a development of the intelligent advisory system for plastics products design to support designer with advice at product development process. The basic idea is to build and apply a knowledge base about plastics materials, related production processes and corresponding design guidelines. The major scientific challenge behind this goal is related to data mining and knowledge discovery. Knowledge acquisition is expected to be the most complex and time consuming part of the research to build the knowledge base, where domain knowledge and data will be collected, organised and properly encoded.

The intelligent system mentioned here is expected to be a major acquirement for many small and medium sized enterprises, as with its application even single designers with less experience will be able to achieve optimal design solutions. The intelligent system will replace the expert team to a great extent so they will be able to focus on new technology development and knowledge dissemination.

\section{Design for $\mathrm{X}$ and knowledge-based engineering}

In today's consumption oriented society consumers' requests and wishes have to be considered as basic guidelines along with product specification as early as in concept phase of design process. Even successful designers have difficulties when linking all the factors and reaching compromises between them. The methodology called "Design for $X$ " (DFX) is extensively applied in engineering practice. "X" 
deputizes many quality criteria like appropriateness for manufacturing, assembly, maintenance, service, etc. [1]. Design for manufacturing (DFM) is mostly used in production process by new product development team as it directly refers to manufacturing process and its costs. All members of the development team as well as outside experts need to contribute their part of expertise to enable the effective DFM practice that leads to low manufacturing costs without sacrificing product quality.

DFX methodology needs to be considered in development of the computer aid for supporting specific design aspects, such as design of plastics products. The second but any less important methodology for our research work is KnowledgeBased Engineering (KBE), which is founded on Artificial Intelligence (AI).

$\mathrm{AI}$ is a branch of computer science that is concerned with automation of intelligent behaviour [2]. The Artificial Intelligence (AI) applications to design are generally concerned with studying how designers apply human intelligence to design, and with trying to make computer aids to design more knowledgeable. These applications are based on representation of heuristic knowledge (which is less easy to express) as mathematical approach is not appropriate in this case. As mentioned, the part of AI that is particularly concerned with the development of such representations are known as expert systems, or more generally knowledge-based systems, often also intelligent computer systems [3].

$\mathrm{KBE}$ is founded on knowledge base, which is existential for intelligent system functionality. KBE is an engineering method in which knowledge about the product, e.g. the techniques used to design, analyse, and manufacture the product, are stored in the knowledge base or product/process model. The model represents the engineering intent behind the geometric design. It contains the attributes of the product such as material type, functional constraints, geometry etc.

Although AI technology is still subject of extensive research and development, many successful AI applications in real-life domains already proved the usefulness of these technologies when dealing with nondeterministic problems that cannot be treated adequately by using conventional approaches, unless the user is possessed of special skills and experience. Engineering design process is certainly one of the domains that very much fit into this scope.

\section{State-of-the-art}

It is hard to imagine a modern design process without using a computer. In fact, CAD is so extensively applied that in many companies all design work is done using these software tools. Yet, there is a body of opinion that the benefits of applying $\mathrm{CAD}$ are below expectations. We believe the reason for this lies in the fact that the existing CAD systems are still not yet adequate as a proper aid to the designer in the design process of a new product. The way in which it is hoped to overcome this bottleneck is to increase the intelligence of CAD systems [4]. 
Existing CAD systems represent a present state-of-the-art in computer support to design process. Some of the systems were already upgraded with intelligence in some technical professional fields. Significant improvement of reliability and effectiveness in performing various engineering tasks was perceived. AI applications are not only a subject of extensive research and implementation but today's reality. Proceedings of the international scientific conferences "AI in Design", edited by J.S. Gero [5], constitute a good collection of papers related to this area.

Design data are not always well formulated, and almost never complete. Experienced designers can deal with such data reasonably easily, while even the most "intelligent" programs have great difficulties. Designers are also reluctant to assign responsibility for decisions to computer programs, no matter how competent they may appear. One can also argue that encoded design knowledge does not allow designers to express their creative ideas. This is even more important in some specific design domains that have their specific constraints and criteria and therefore require specific approach in design process. For all these reasons, computer (intelligent) support to specific design aspects, including those that are subject of the proposed research, is still quite limited and therefore insufficient.

\subsection{Design of plastics products}

In today's world, designer should follow quite extensive list of basic steps and procedures to produce a world-class product [6]. Within this process, different design decisions have to be taken, like choosing the appropriate material, production process, tooling, equipment, services, etc. The single designer is not able to reach all the correct decisions so the consultations with experts of different expertise are of high importance. Furthermore, design with polymers requires more involved and upfront engineering approach than ever before. This is why the intelligent computer support to plastics design is essential.

Yet, quite many AI applications have been reported in this particular field of design. In 1996, Rapra Technology Ltd. [7] claimed to lunch first ever knowledge based system for the plastic industry. Most of the later AI applications were addressing separate parts of design process, i.e. the selection of specific materials, such as ceramic [8], or the use of special manufacturing and corresponding tooling processes, where injection moulding is far the most popular [9, 10]. On the other hand, no serious attempt is recorded to develop the intelligent advisory system for supporting plastics products design process as a whole. Therefore, our research presented in this paper represents a novel contribution to this important technical field. 


\section{Problem presentation}

In present time, each product is exposed to the competitive struggle on the market, where success can be expected only for universally optimal design solutions. Consequentially, specific design aspects like design of plastic products are becoming increasingly important and are not left behind in correlation with functionality and economic efficiency. Every competent enterprise with intention to take the leading position in its branch is aware of this fact and is opened for any process application in order to achieve that goal. Therefore, it is no surprise that CAD is so extensively applied.

In spite the fact that modern CAD tools are very strong in graphic presentation, the limitations at providing design recommendations to the user are becoming more and more obstructive. Design projects normally originate in form of problem statement provided to the designer by the company management. These problem statements set a goal, some constraints within which the goal must be achieved, and some criteria by which a successful solution might be recognised. It is usually possible to improve the initial definition of the problem. Yet, many design constraints and criteria still remain unknown and the existing CAD approaches are not able to help designer in dealing with uncertainty and inconsistencies. Thus, the quality of design solution depends mostly on the designer's skill and experience.

Designers face many dilemmas linked with various aspects of the product. Compromises have to be considered at every design step. In order to create as optimal compromises as possible, designers have to possess wide range of knowledge and have to be aware of all influential parameters, or alternatively a team of experts in various fields has to collaborate in development process [11].

The designer often stands at the crossroads as product specifications and customer requirements are very much contradictive with specific design issues like how to produce, to assemble, to maintain or to service the product. In this case, DFX methodologies can be very helpful but often rather not sufficient.

Figure 1 shows product development process, following four phases of design (upper part of the Figure 1): task clarification, conceptual design, embodiment design and detail design.

The designer is progressing through design phases relying on personal and expert team's knowledge. As the alternative to the extensive expert team, which is often not on disposal, we propose a supplement to the existing CAD tools in form of the intelligent advisory system for supporting plastics product design described in this paper. Parallel with research in field of intelligent support to plastics design, the intelligent systems for supporting ergonomic and aesthetic design are also subject of development in our laboratory [12].

It is anticipated the proposed intelligent supporting system to comprise several intelligent modules, each based on the knowledge base for specific design aspect. The lower part of the Figure 1 shows the idea of the proposed intelligent system 


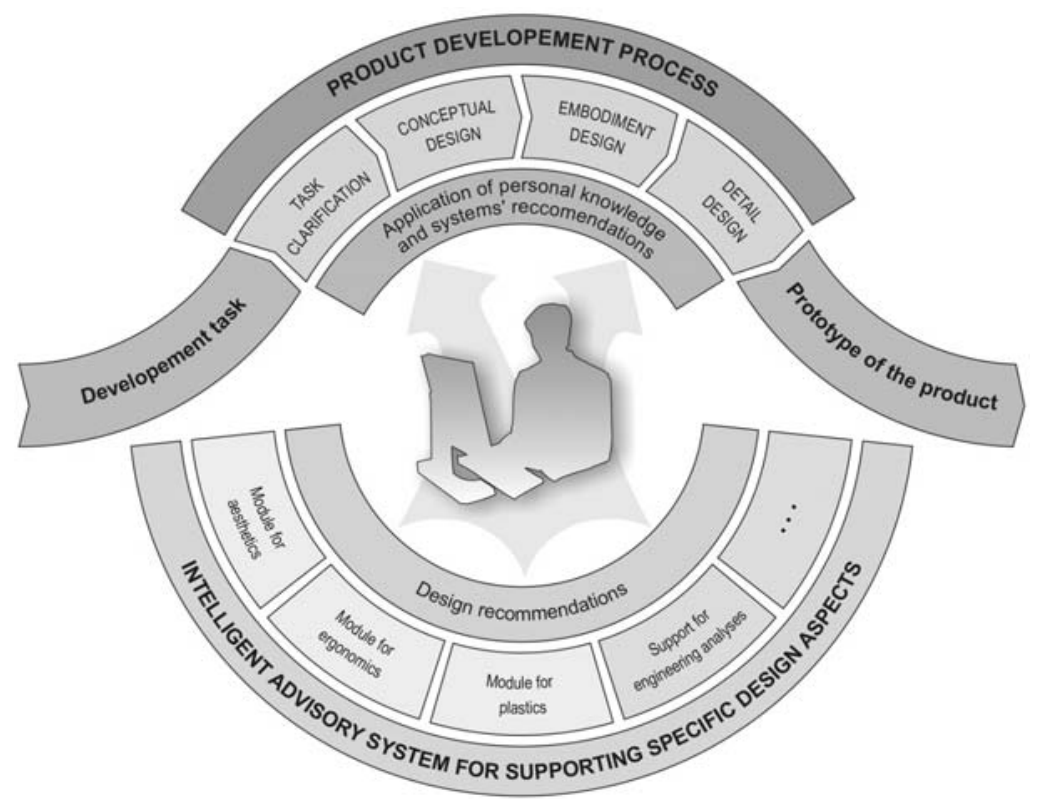

Figure 1 Product development process supported with intelligent advisory system

also presenting the intelligent module for supporting engineering analyses, which was already developed in our research group and gave promising results.

Above all, the main purpose of the intelligent system is to support inexperienced designer by qualified stream of advices in terms of design recommendations and guidelines for specific design aspects. Using this intelligent computer support, the designer will be able to use the "knowledge" encoded in the knowledge base of the system to follow basic steps of design and to find optimal design solutions easier and faster.

\section{Intelligent advisory system}

To consider the world without plastics is today almost impossible to imagine. Only the most innovative enterprises, which are investing in development and are keeping up with their competition, can be successful. In engineering practise designers usually decide mostly upon well-known tested materials like metals, wood and ceramics. In the present time, this kind of materials can be substituted with others, more suitable for the certain type of product. Plastics are one of those alternative materials, as they can offer optimal characteristics for noticeable lower costs. Due to the assortment of polymers (over 120 thousand) available on the market, the expert working team should be numerous, while planning the new 
product. Consequentially, the designer is forced to rely upon knowledge and experience of the working partners.

According to previous writing some major problems of plastics product development process can be outlined and summarised in two basic groups:

1. New materials are not adequately represented in engineering practise due to insufficient knowledge about new materials and related characteristics, as well as due to traditional use of other more familiar materials.

2. Information about plastics, their properties, related machining processes and matching design recommendations are not properly collected and organised for engineering use.

Development of the intelligent advisory system for plastics design will be clearly an acquirement to the great extent, as engineers will finally be able to get some professional recommendation how to deal with some specific design aspects in plastics product design process. In this manner, they will be able to select the material best suited to the purpose of the product without dependency on their limited experience, creativity and product performance requirements.

\subsection{KBE and DFX in plastics design}

Design is a very complex process. One of the crucial decisions that need to be made within this process is also the selection of the material for the new product. Material selection is always affected by basic demands, like application manner and additional factors, like supplier recommendation, own experience, etc. Moreover, the designer also has to anticipate the production process, semi-product or product assembly, maintenance of the part and environmental component, which becomes especially important due to the pollution of the planet. For all this reasons, DFX methodology has to be considered very thoroughly, especially at plastics product design process. However, designer still can not expect any adequate help in form of recommendation or guidelines when material or technological process has to be selected to achieve maximal quality at minimal costs.

In order to overcome this bottleneck, KBE techniques need to be considered along with DFX methodology, when developing the intelligent advisory system for plastics product design. Figure 2 presents the basic idea and is visually divided

in three components: input, intelligent module and output. Input containing customer's requests, wishes, and technical criteria is general for any design problem, the same as the output with selected material and some design and production guidelines. The intelligent module for plastics product design represents a new component in design process. The preliminary condition for the knowledge-based support to plastics product design process is the adequate knowledge base containing related, well organized DFX knowledge, relations and data. 


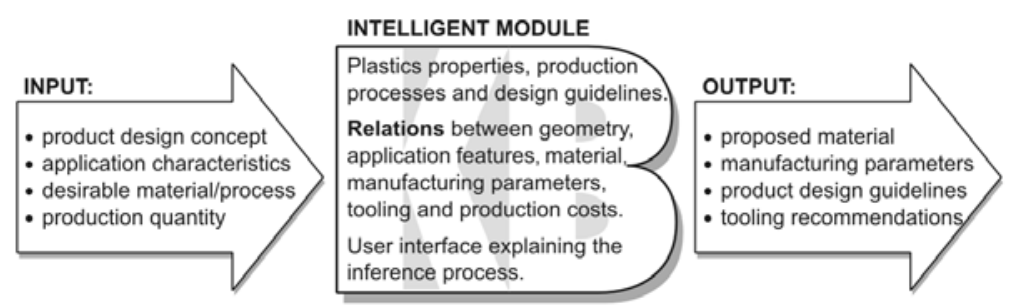

Figure 2 Knowledge-based design of plastics products

It is evident that expert support in decision-making process is essential for almost every designer to perform design of plastics products successfully and efficiently. Thus, we decided to develop an intelligent advisory system to support this important design issue. Above all, the expected development methods include a combination of basic design knowledge with special domain expertise in field of plastics. The knowledge base will contain rules related to selection of the modern plastic materials and correlated manufacturing processes, as well as special guidelines and recommendations for designing plastics products. Different approaches to knowledge acquisition and the appropriate formalisms for the presentation of the acquired knowledge within the computer program will be of special importance. The potential of transparent and modular IF-THEN rules is planned to be compared with more flexible knowledge presentation systems such as fuzzy logic.

Figure 3 shows comparison between conventional approach of plastics product design and design process supported with the intelligent advisory system.

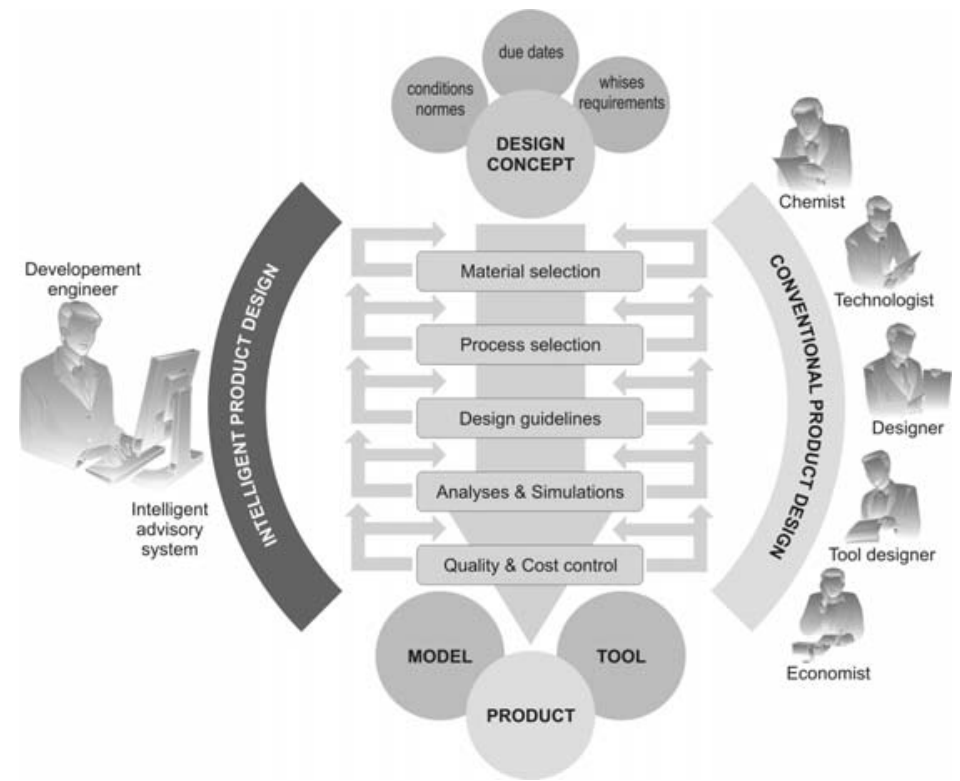

Figure 3 Design process with intelligent system support vs. conventional approach 
Requirements, wishes, conditions and due dates are passed to designer either from the management or direct from the customer. Designer's responsibility is to design the model, tool or finished product by carrying out the whole development process. Thus, designer should consult with experts like technologist, chemist, tool designer and economist, who are able to deliver their expert knowledge as this is the only way to achieve performance of optimal solution.

As the alternative to the expert team (Figure 3), some tasks like choosing the material and process, presenting the design guidelines, performing the analyses and monitoring the quality and costs, can be supported by applying the intelligent advisory system proposed here.

In present practice design process is sometimes still successive. The customer provides the designer with input data where the requests frequently predominates technical criteria. Therefore, the designers and technologist are often handicapped when trying to enhance the quality of the product or a process. In such case, the need for intelligent computer support is also very expressive.

The intelligent system will be developed in form of consultative advisory computer tool to be used interactively. The main goal for the system is to apply domain knowledge, relations and experiences from the knowledge base of the system in complex reasoning procedure leading to qualified design recommendations.

In order to enable transparent and efficient system application, the user interface will be developed with a special attention. Regarding the type of input and output data, two different application modes are anticipated. Guided mode (question and answer) will be used mostly at the beginning, when first set of parameters has to be presented to the system. During data processing phase, the system may present additional questions or ask for more parameters. In this case, guided and graphic mode will be used to present the problem to the user. In final phase, the solution will be presented in graphic mode if possible.

\section{Conclusions}

Knowledge and experience of design experts are of crucial importance for plastics product design process. Thus, young inexperienced designers have many difficulties when facing the challenge to make crucial decisions within complex design process. With development of the proposed intelligent advisory system, knowledge and experiences will be collected, systematised and arranged in the modulebased knowledge base.

KBE techniques are already extensively applied in developed world mostly in military, airplane and automotive industry. Small and medium sized enterprises are also aware of KBE advantages but do not have enough human and financial resources for implementation of those techniques in development process. Consequentially, their competitiveness on the market is aggravating as for higher development costs and related product price. The intelligent advisory system for plas- 
tics design will help them to achieve their business goal: »maximal quality at minimal costs « less experience-dependent and with higher efficiency. The experts working on one project will be contracted and the team members will be able to dedicate to the new technologies and dissemination of personal knowledge.

The research presented in this paper is a part of the broader research activities, performed by members of our laboratory. The aim of these activities is to develop intelligent advisory systems for supporting product realization process. Our recent results in this research field are two prototypes of the intelligent systems, first to support finite element selection process [13], and the other to support design optimisation considering the results of the structural engineering analysis [14].

The proposed system is also meant to be used in education for the students of engineering and product design as typical representatives of inexperienced designers. In this case, the important feature of the intelligent systems that usually have the ability to explain the interface process will be especially welcome.

\section{References}

1. Huang G.Q. (Ed): Design for $\mathrm{X}$ - concurrent engineering imperatives. Chapman \& Hall (1996).

2. Luger G.F., Stubblefield W.A.: Artificial Intelligence and the Design of Expert Systems. The Benjamin/Cummings Publishing Company Inc, Redwood City (1989).

3. Turban E., Aronson J.E., Liang T.P.: Decision Support Systems and Intelligent Systems. Prentice Hall, 7th edition (2004).

4. Finger S., Ttetsuo T., Martti M.: Knowledge Intensive Computer Aided Design. Kluwer Academic Publishers (2000).

5. Gero J.S. (Ed): Artificial Intelligence in Design '02. Springer (2002).

6. Gordon M.J.: Industrial Design of Plastics Products. John Wiley \& Sons (2003).

7. Rapra Technology Ltd.: Knowledge based expert system for the plastics industry. In: Materials \& Design. Vol. 17, No. 4, pp. 227 (1996).

8. Sapuan S.M., Jacob M.S.D., Mustapha F., Ismail N.: A prototype knowledge-based system for material selection of ceramic matrix composites of automotive engine components. In: Materials \& Design. Vol. 23, pp. 701-708 (2002).

9. Mok C.K., Chin K.S., Hongbo L.: An Internet-based intelligent design system for injection moulds. In: Robotics and Computer-Integrated Manufacturing. Vol. 24, Issue 1, pp. 1-15 (2008).

10. Wang K.K., Zhou J.: A Concurrent-Engineering Approach Toward the Online Adaptive Control of Injection Moulding Process. In: CIRP Annals - Manufacturing Technology. Vol. 49, No. 1, pp. 379-382 (2000).

11. Clarkson J., Eckert C. (Eds): Design process improvement - a review of current practice. Springer (2005).

12. Kaljun J., Dolšak B.: Computer Aided Intelligent Support to Aesthetic and Ergonomic Design. In: WSEAS Transactions on Information Science and Applications. Vol. 2, No. 3, pp. 315-321 (2006).

13. Dolšak B.: Finite element mesh design expert system. In: Knowledge-based systems. Vol. 15, No.5/6, pp. 315-322 (2002).

14. Novak M., Dolšak B.: Intelligent computer-aided structural analysis-based design optimisation. In: WSEAS transactions on information science and applications. Vol. 3, No. 2, pp. 307314 (2006). 